\title{
MedienPädagogik
}

Zeitschrift für Theorie und Praxis der Medienbildung

Jahrbuch Medienpädagogik 4.

Zweitveröffentlichung aus: Jahrbuch Medienpädagogik 4. (2005) Wiesbaden: VS Verlag für Sozialwissenschaften. Hrsg. v. Ben Bachmair, Peter Diepold und Claudia de Witt.

\section{Plädoyer für Offene Lernumgebungen}

\author{
Rolf Schulmeister
}

Ich möchte eingangs drei Behauptungen oder Thesen aufstellen, und vielleicht gelingt es mir, sie im nachfolgenden Aufsatz durch einige Argumente plausibel zu machen:

- Lehren und Lernen unterscheiden sich nicht danach, ob sie in Präsenzlernphasen oder in virtuellen Lernumgebungen stattfinden. Diese Aussage gilt auch für Lehrmethoden und Lernmethoden.

- Einige Lehr-Lernmethoden scheinen allerdings für virtuelle Lernumgebungen geeigneter zu sein als andere Methoden. Zu den für virtuelles Lernen eher geeigneten Lehr-Lernmethoden zähle ich die sog. „offenen Lernumgebungen“.

- Virtuelles Lernen erreicht erst dann eine dem Präsenzlernen adäquate Qualität, wenn es einen hohen Grad an Aktivität erlaubt und/oder eine intensive Kommunikation ermöglicht.

Eine Unterscheidung muss ich allerdings vorab einführen, die den Geltungsbereich dieser Thesen etwas eingrenzt: Ich unterscheide eLearning-Umgebungen danach, ob sie einen relativ standardisierten Wissenskanon anbieten und damit ein individuelles Lernen ermöglichen oder ob sie anfänglich keinen Wissensbestand vorgeben, sondern einen projektorientierten Ansatz verfolgen und auf die Bildung sog. Wissensgemeinschaften zielen. Mit anderen Worten: eLearning-Umgebungen variieren vom individuellen Selbststudium bis hin zu kooperativen Lern- und Wissensgemeinschaften (Schulmeister 2003, S. 163-187), von einem asynchronen Lernen mit digitalen Lernobjekten bis hin zu synchronen Diskussionen mit anderen Studierenden. 


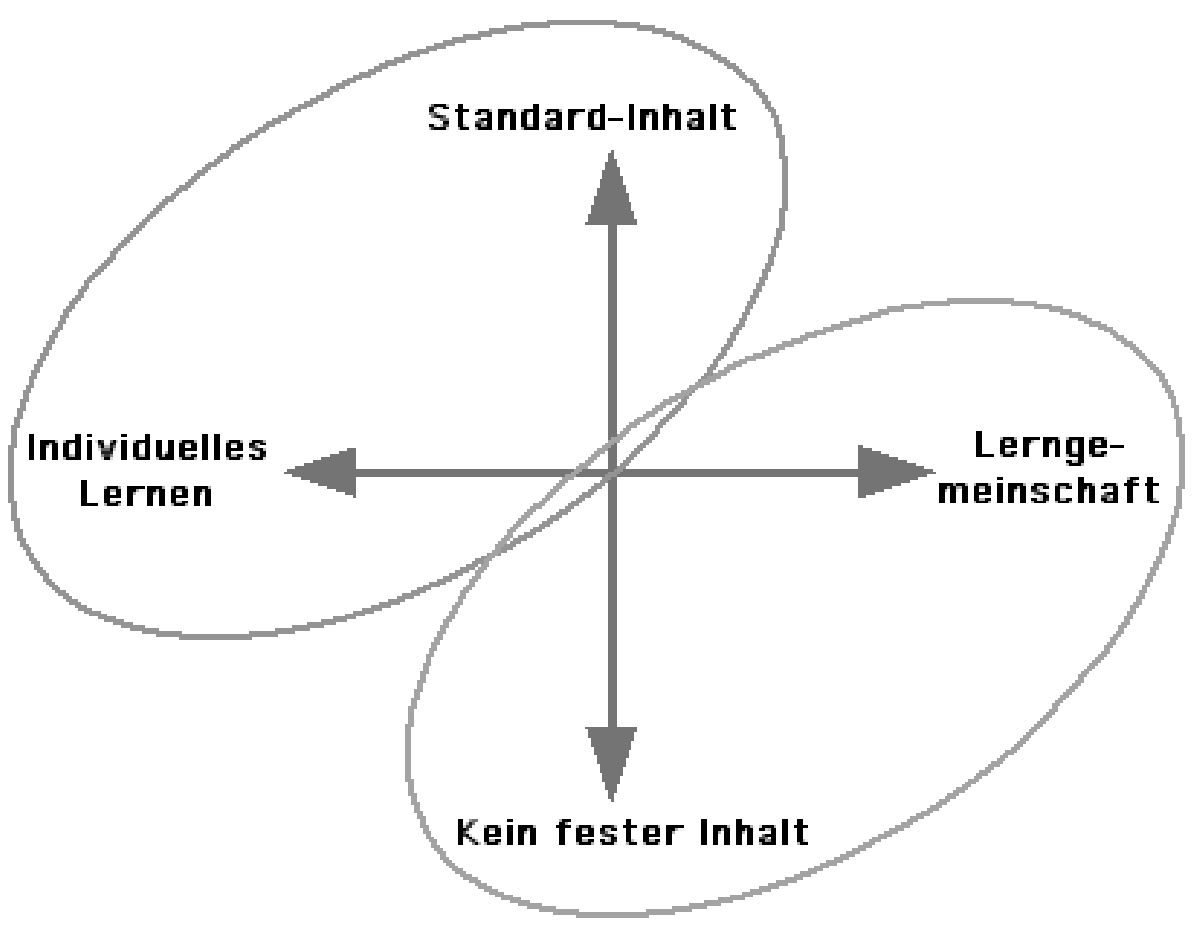

Abb. 1: Zwei Typen virtueller Lehre.

Zwischen den Extremen dieser Skalen befinden sich alle möglichen denkbaren Mischformen virtueller Lehre, virtuelle Vorlesungen, virtuelle Arbeitsgruppen mit klaren Aufgaben, virtuelle Seminare mit geringen synchronen Kommunikationsanteilen, selbständige Projektgruppen mit hohen kommunikativen Anteilen. Warum treffe ich diese Unterscheidung?

Nicht alle Aussagen, die zum eLearning gemacht werden, gelten für die beiden Typen von eLearning gleichermassen. Für die Wissens- oder Lerngemeinschaften sind Fragen der Kommunikation, der Kooperation und der Moderation vorrangig, während für Selbstlernumgebungen Lernziele, Lerninhalte, Methoden und Mediengestaltung wichtiger sind. Ich werde mich im Folgenden mit meinen Aussagen auf Lernumgebungen beziehen, die mit vorgegebenen Lernobjekten arbeiten und diese den Studierenden zum Selbststudium anbieten oder in mehr oder minder kommunikativen Unterrichtsarrangements verarbeiten.

\section{Zwei Beispiele}

Welche Qualitäten das Lernobjekt in virtuellen Lernumgebungen haben sollte, möchte ich an zwei Lernprogrammen demonstrieren, die zum Erlernen der Gebärdensprache für unsere hörenden Studierenden entwickelt wurden. 
Das erste Beispiel ist ein multimediales Programm zum Erlernen der Gebärdensprache mittels natürlicher Dialoge und das zweite Beispiel ist ein Programm für die Konstruktion gebärdensprachlicher Äußerungen, das einen Avatar als Tutor einsetzt.

Wir hatten vor drei Jahren ein multimediales Programm zum Erlernen der Deutschen Gebärdensprache entwickelt (Metzger, Schulmeister und Zienert 2000). Wir haben das Lernprogramm „Die Firma“ genannt, weil die elf Lektionen in einer Firma spielen, in der Gehörlose zusammen mit Hörenden an Bauprojekten arbeiten. Alle elf bestehen aus authentischen Szenarien mit natürlichen Dialogen. Die Aufgabe der Studierenden ist es, durch Analyse der Dialoge und durch den Vergleich des situativen Gebrauchs von Gebärden im Kontext mit ihrer Zitationsform im Lexikon die Grammatik der Gebärdensprache selbständig zu erschließen.

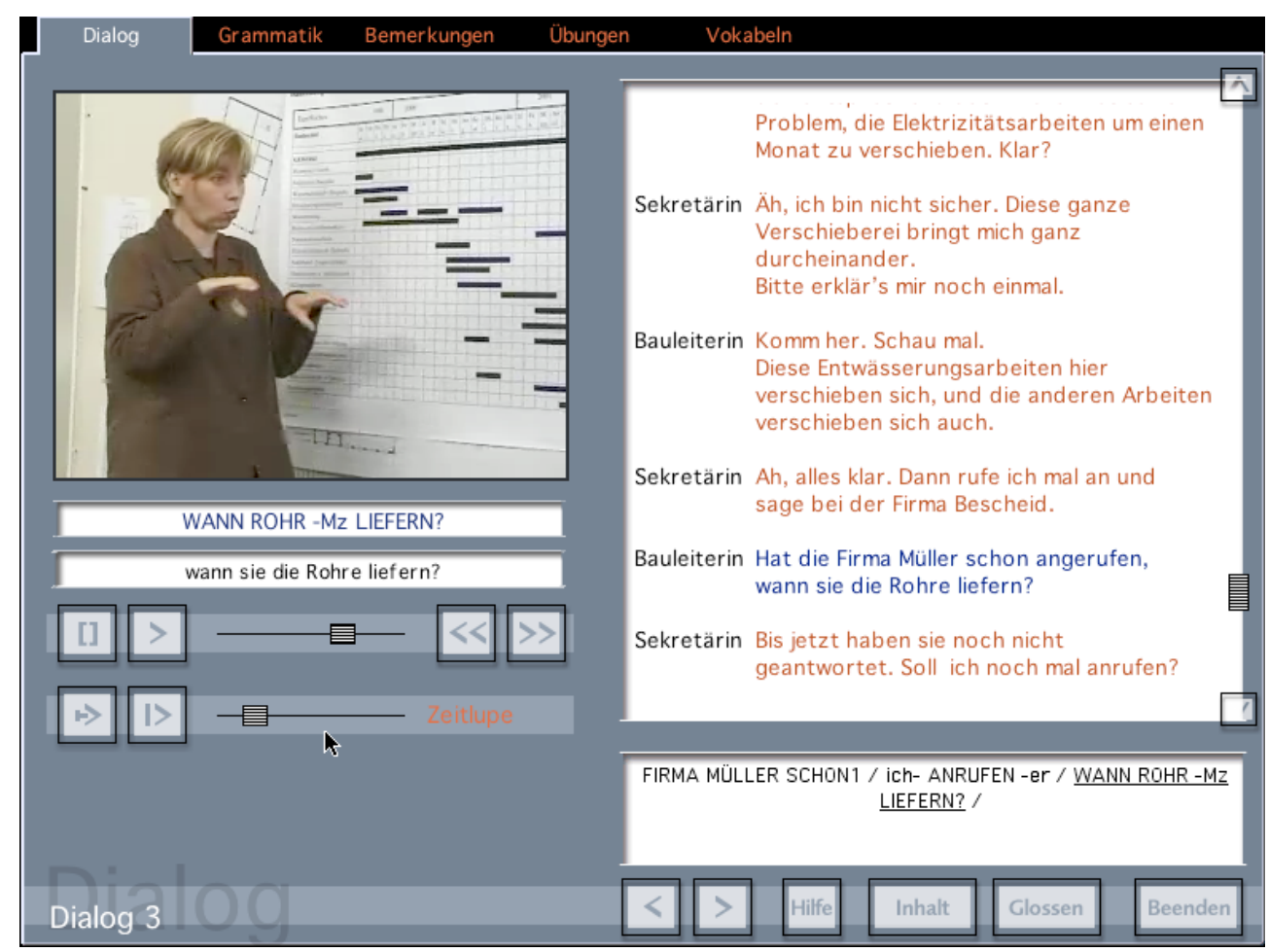

Abb. 2: Die Firma II: Dialog im Lernprogramm zur Gebärdensprache.

Die erste Version des Programms „Die Firma“ enthielt pro Lektion auch Übungen, mit denen die Studierenden ihre rezeptive und produktive Sprachkompetenz testen konnten. Wir hatten damals jedoch keine Methode gefunden, das Programm die Sprachkompetenz der Benutzer überprüfen zu lassen und dazu Rückmeldung geben zu können. In einer neuen Version „Die Firma II“ (Metzger, Schulmeister und Zienert 2003) haben wir jetzt Spiele als interaktive Lern- 
objekte eingebaut, in denen die Studierenden ihr Verstehen der Gebärdensprache in Handlungen umsetzen müssen und vom Programm Rückmeldung dazu erhalten können. In den Dialogen der einzelnen Lektionen geht es um folgende grammatische Prinzipien der Gebärdensprache:

- Einsatz von Klassifikatoren und INDEX-Gebärde

- Die Gestaltung von Zeitlinien und Zeitpunkte-Markern

- Ausdruck der Perspektive und der Wechsel der Perspektive

- Die Benutzung von Formdeskriptoren

Die Übungen bestehen aus interaktiven zwei- oder dreidimensionalen Lernobjekten. Im linken Fenster erläutert der Lektor die Aufgabe in Gebärdensprache. Auf der rechten Seite kann der Studierende in Konstruktionshandlungen umsetzen, was er aus der Anweisung verstanden hat. Sofern er die Aufgabe erfolgreich umsetzt, erhält er eine positive Rückmeldung vom Lektor. Hat er die Anweisung nicht korrekt verstanden, wird er auch die Aufgabe nicht lösen können. In dem Fall erhält er entsprechende Rückmeldungen durch Animationen und Hinweise vom Lektor.

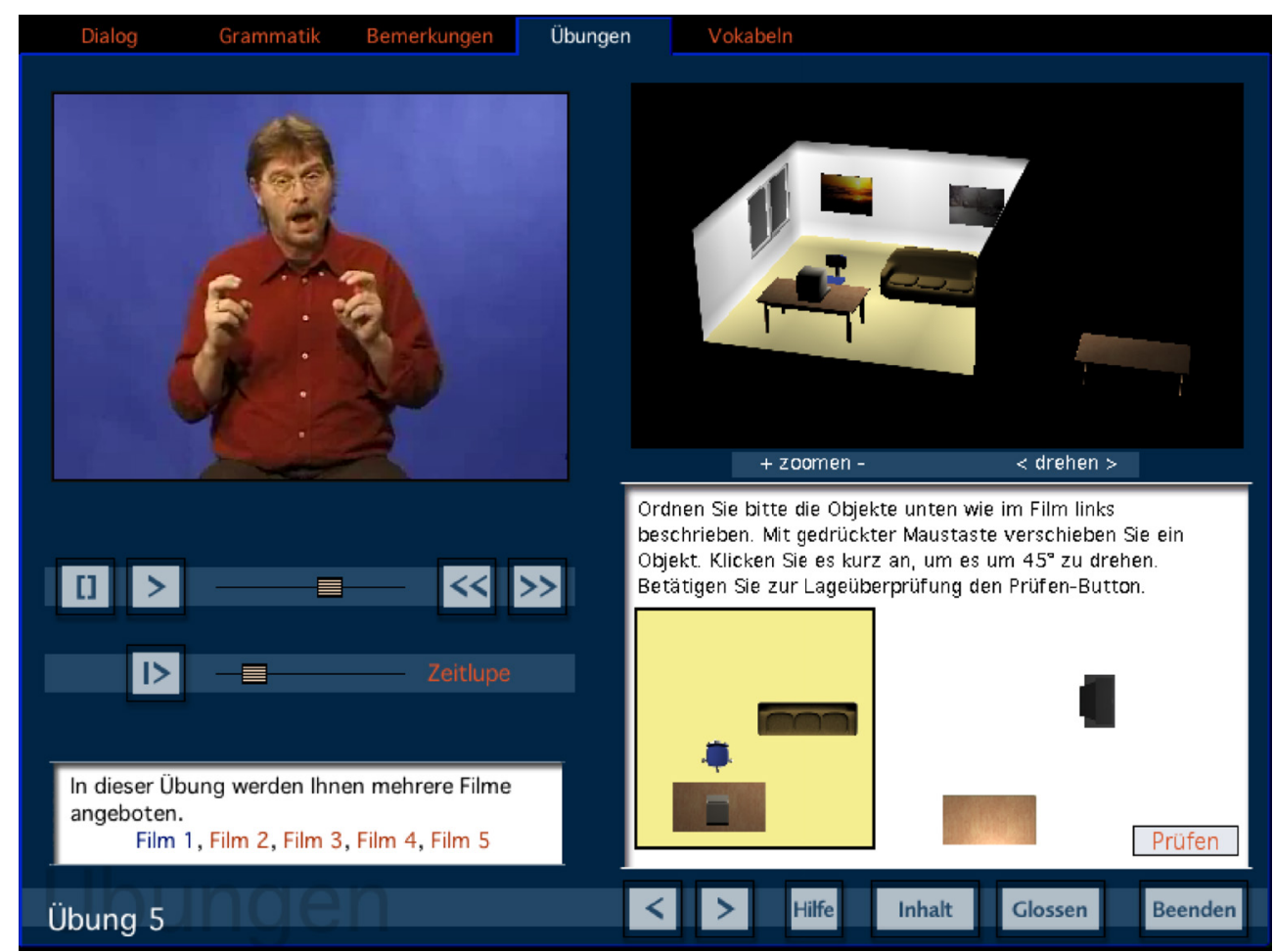

Abb. 3: Die Firma II: Interaktive Übung: Arrangement von Möbeln.

Die interaktiven Übungen beinhalten folgende konstruktive Aufgaben:

- In Lektion 2 müssen verschieden geformte Räume in unterschiedlich geformten Stockwerken eines Gebäudes richtig arrangiert werden 
- In Lektion 4 müssen unterschiedlich geformte Räume mit Möbeln und Elektroanschlüssen etc. ausgestattet werden

- In Lektion 5 sollen Aufgaben in einer Stadt erledigt werden. Der Studierende lenkt ein Auto durch die Straßen einer Stadt (2D-Aufsicht von oben) und muss dabei bestimmte Info-Punkte anlaufen (Post, Universität, Bank etc.)

- Fahren in einem 3D-Labyrinth.

Das Programm kann die korrekte Erledigung der Aufgaben durch Verfolgung der grafischen Interaktion abprüfen und Feedback dazu geben. Da es sich bei der Gebärdensprache um eine Bewegung im Raum handelt, boten sich grafische Manipulationen in 2D und 3D als geeignetes Konzept für die Konstruktion der interaktiven Übungen an.

Weitaus komplizierter war es jedoch, eine geeignete Methode für ein Feedback zur produktiven Sprachkompetenz zu finden. Die Entwicklung einer Methode zum Überprüfen der produktiven Sprachkompetenz hat uns einige Kopfschmerzen bereitet. Im Projekt ViSiCAST wurde ein „Signing Tutor“ entwickelt (Popescu, Hong, Schmaling, Hanke, Schulmeister et al 2002). Dieser Tutor ist ein Avatar namens Visia, der Gebärdensprache auf dem Bildschirm synthetisch generiert, wobei Visia durch eine linguistische Maschine gesteuert wird.

Die Tutorin Visia ist ein Demonstrator der Übersetzungstechnologie von ViSiCAST. Ihre Bewegungen werden nicht durch motion capturing generiert, sondern die Gebärden von Visia werden synthetisch generiert. Die Gebärden von Visia wurden mit HamNoSys 4 notiert und werden vom Programm in SiGML umgewandelt, einer XML-Variante, die die Animation steuert.

Der Signing Tutor ist zum Lernen der Gebärdensprache entwickelt worden. Der Tutor ist eine hochinteraktive Umgebung, in der die Studierenden probieren können, eigene Aussagen oder Sätze in Gebärdensprache zu konstruieren. Der Tutor kann auch selbständig richtige und falsche Sätze bilden, mit deren Hilfe die Studierenden ihre produktive Sprachkompetenz testen können.

Der Einsatz des Avatars hat mehrere Vorteile:

- Die Studierenden können den Avatar rotieren und zoomen und so die dreidimensionalen Bewegungen der Gebärdensprache besser verfolgen

- Die Studierenden können selbst gebärdensprachliche Äußerungen konstruieren

- Der Avatar kann vom Studierenden angezielte Äußerungen geringfügig variieren, um die Sprachkompetenz der Studierenden zu testen

- Die Studierenden können gemäß eigener Lerngeschwindigkeit studieren

- Die Studierenden können das Programm unabhängig vom Ort nutzen

- Die Studierenden können die Lernprozesse beliebig oft wiederholen

- Das System unterstützt das Gedächtnis, während die im Präsenzunterricht gemachten neuen Erfahrungen kaum vernünftig memoriert werden können. 
Im Vergleich zu einem multimedialen Lernprogramm mit fest gespeicherten Videosequenzen, wie es in dem Beispiel „Die Firma“ vorliegt, hat der Einsatz eines Avatars weitere Vorteile:

- Der Avatar kann eine unbegrenzte Menge an Gebärden und Sätzen bilden

- Der Studierende kann Beispiele konstruieren, die nicht vorhergesehen wurden und deshalb nicht gespeichert werden konnten

- Man kann den Avatar veranlassen, Fehler zu machen und mit den Fehlern den Studierenden zu testen.

In der Lektion 1 können die Studierenden das grammatische Prinzip der Zahleninkorporation in der Gebärdensprache explorieren. Die Gebärden für Stunden, Tage, Wochen, Monate und Jahre sowie Geldmengen werden mit den Gebärden für niedrige Zahlen zu neuen Gebärden verschmolzen, mit höheren nicht. Es gibt auch Gebärden für Zeitangaben, die nicht mit den Zahlengebärden verschmolzen werden. Das Prinzip der Verschmelzung wird in der Gebärdensprach-Linguistik als Inkorporation bezeichnet.

Lektion 1: Zahleninkorporation (2)

Demonstration

Diese Demonstration gibt dir Gelegenheit alle Zahleninkorporationen in DGS kennenzulernen.

Gib in das erste Feld eine Zahl zwischen 1 und 9999 ein und wähle im zweiten Feld aus dem Pop-up Menii eine Angabe aus. Visia wird dir die entsprechenden Gebärden dazu zeigen. Gib sowohl Zahlen von 1-10 wie auch Zahlen von 11-9999 ein, um inkorporierte wie auch nicht inkorporierte Kombinationen kennenzulernen.

Ein sorgfältiges Arbeiten mit dieser Demo wird dich gut auf alle folgenden Übungen vorbereiten.

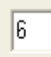

$6 \quad-$ Jahr $\quad-$ Zeigen

\section{(2) 0 (1)}

Abb. 4: Signing Tutor „Visia“ generiert Gebärdensatz. 
In der Lektion 2 können die Studierenden „Richtungsverben“ mit Gegenständen verbinden (z.B. ich gebe ihm ein Buch), wobei die Gegenstände als Klassifikatoren die Handform des Verbs modifizieren. Dieses Prinzip gilt allerdings nicht für alle Objekte. Der Studierende kann ein Verb und ein Objekt wählen und die Richtung (Adressat, indirektes Objekt) bestimmen.

\section{Lektion 2: Richtungsverben (2) \\ Demonstration}

Visia gibt dir Gelegenheit alle in der Lektion vorkommenden bidirektionalen Verben kennenzulernen. Anhand des Pop-up Menüs kannst du ein bidirektionales Verb auswählen. Ggf. hast du im zweiten Feld auch die Möglichkeit ein zugehöriges Sachobjekt zu bestimmen. Mit der Maus kannst du dir aus der Palette rechts einen Pfeil aussuchen und in das Schaubild einfuigen; die Pfeilspitze zeigt das indirekte Objekt und das Pfeilende das Subjekt an. Ein sorgfältiges Arbeiten damit wird dich auf alle folgende Übungen gut vorbereiten. Achte darauf, welche bidirektionalen Verben keine Pfadbewegung haben und bei welchen Verben eine Klassifikatorhandform inkorporiert wird.
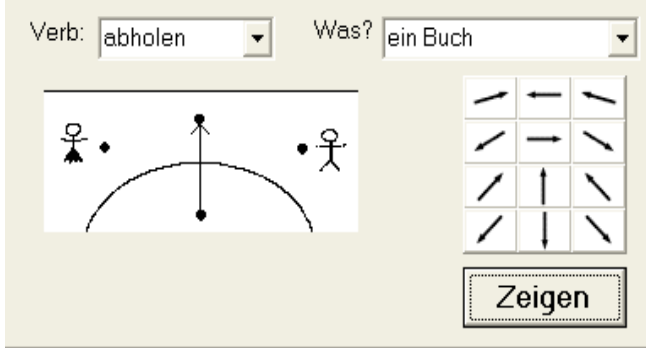

\section{(2) 0 (1)}

Abb. 5: Signing Tutor: Generierung von Richtungsverben.

Der Signing Tutor erlaubt konstruktive Sprachhandlungen und kann auch als Testwerkzeug genutzt werden, indem man ihn veranlasst, falsche und halbfalsche Lösungen zu präsentieren. Die Beispiele aus der Gebärdensprache machen deutlich, wie hochinteraktive Übungen und Feedback zu anspruchsvollen Lernobjekten verbunden werden können. Zugleich dürften sie deutlich gemacht haben, dass es keine leichte Aufgabe ist, hoch-interaktive Lernobjekte für virtuelle Lernumgebungen zu entwickeln, die Rückmeldung zu Lernprozessen geben können.

\section{Gemeinsame Charakteristika der beiden Lernumgebungen}

Was unterscheidet die geschilderten Beispiele von klassischen instruktionalistischen Umgebungen? Warum rechtfertigen sie die These, dass sich für elearning-Umgebungen, die mit Wissens- und Lernobjekten arbeiten, offene Lernumgebungen als didaktisches Gestaltungsprinzip an bieten. Was sind of- 
fene Lernumgebungen? Offene Lernumgebungen kann man mit Elen und Lowyck beschreiben als

„powerful learning environments focus on providing a supportive ecological system that is less structured and less directive, that encompasses learner-controlled tools for the acquisition of knowledge and skills, and that attempts to integrate both tools and coaching strategies in collaborative learning environments“ (Elen/ Lowyck et al. 1999, S. 192).

Welche Modelle offener Lernumgebungen sind bekannt? Ohne Anspruch auf Vollständigkeit sind dies zum Beispiel folgende Methoden, die entweder auf der Grundlage kognitionspsychologischer oder konstruktivistischer Lehr-Lern-Konzepte entstanden sind:

\begin{tabular}{l|l}
\hline Anchored Instruction & $\begin{array}{l}\text { Cognition and Technology Group at Vanderbilt } \\
1993\end{array}$ \\
$\begin{array}{l}\text { Apprenticeship Learning } \\
\text { Legitimite peripheral participation }\end{array}$ & $\begin{array}{l}\text { Collins 1998 } \\
\text { Reciprocal Teaching } \\
\text { Fallbasiertes Lernen (case-based study) }\end{array}$ \\
$\begin{array}{l}\text { Problemorientiertes Lernen (problem-based } 1991 \\
\text { learning) }\end{array}$ & $\begin{array}{l}\text { Schmidt 1989; Gräsel, Mandl et al 1992; Jonas- } \\
\text { Lernen mit Simulationen }\end{array}$ \\
$\begin{array}{l}\text { Entdeckendes Lernen } \\
\text { Forschendes Lernen }\end{array}$ & $\begin{array}{l}\text { Goodyear 1991; de Jong \& Njoo } 1992 \\
\text { Bruner, 1961; Shulman \& Keislar } 1966 \\
\text { Bundesassistentenkonferenz 1970 }\end{array}$ \\
\hline
\end{tabular}

Wodurch zeichnen sich offene Lernumgebungen aus? Offene Lernumgebungen setzen in der Regel einen Lernanlass oder Rahmen für den eigentlichen Lernprozess. Hier kann es sich um ein Szenario, ein Problem, eine Praxis, eine Umgebung zum Explorieren oder ein kognitives Werkzeug für die Konstruktion von Simulationen oder anderen Objekten handeln, wobei manche problemorientierten Ansätze z.B. in der Medizin eine prozedurale Struktur anbieten, die sich an der Heuristik des Problemlöseprozesses in der Praxis orientiert (s. Schulmeister 2002).

Der Rahmen oder Lernanlass für die Lernumgebung beruht möglichst auf authentischen Situationen oder Szenarien, die geeignet sind, die Motivation der Lernenden anzusprechen und den sozialen oder natürlichen Kontext für die Lerngegenstände zu repräsentieren. Innerhalb des vorgegebenen Rahmens öffnet sich für die Lernenden ein Suchraum für explorative Lernprozesse, in dem sie Handlungen probieren können, die anfangs vielleicht dem Muster von Versuch \& Irrtum folgen, dann aber mit zunehmender Erfahrung einem gezielten Testen von Hypothesen weichen. Wichtig anzumerken ist, dass die Lernenden anfangs zumeist mit naiven kognitiven Konzepten an die Lernobjekte herantreten und diese naiven Konzepte erst im Prozess des Problemlösens in formale wissenschaftliche Konzepte transformieren. 


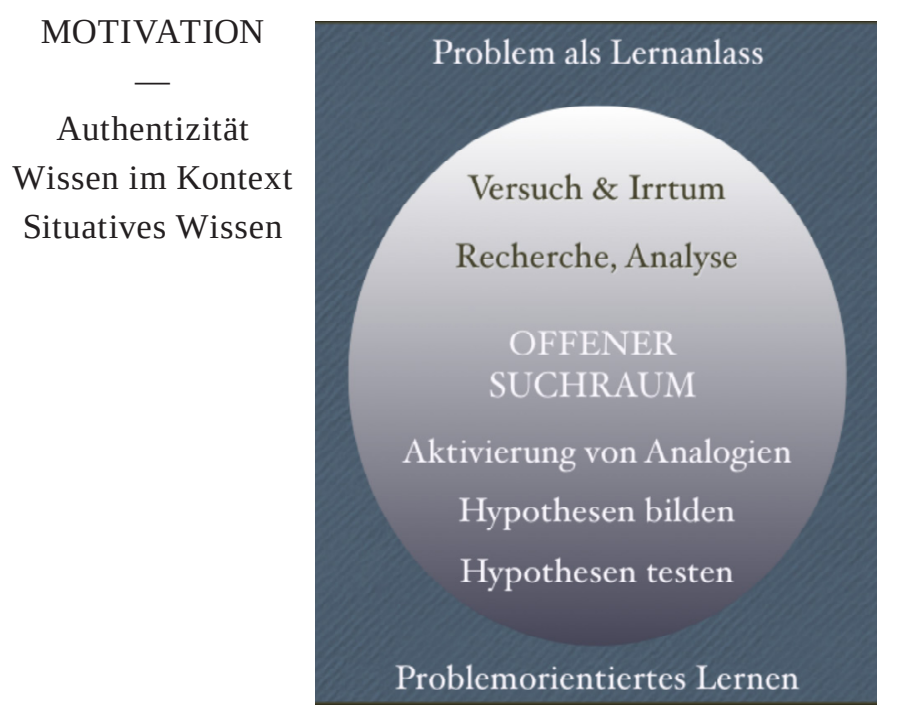

KOGNITION

Naive Konzepte

formale Konzepte

Abb. 6: Szenario und Suchraum.

Diese Art von Lernsituationen geben keine bestimmten Inhalte oder Aufgaben vor, sondern überlassen es den Lernenden, selbst die Ziele und Gegenstände des Lernens zu entdecken und sich das benötigte Wissen im Verlauf des Problemlöseprozesses selbständig anzueignen. Sie folgen dem Konzept des studentenzentrierten Lernens (Jonassen 2000).

Offenes Lernen in authentischen Umgebungen scheint mir die adäquate Methode für Prozesse der Wissenskonstruktion und Konstituierung von Bedeutungen zu sein. Dies gilt allerdings nur unter einer wichtigen Voraussetzung: In offenen Lernumgebungen spielt die Qualität der Lernobjekte eine besondere Rolle, eine Qualität, die durch den Grad an Interaktivität des Lernobjekts konstituiert wird und die durch die Rückmeldung unterstützt wird, die der Lernende durch die Manipulation des Lernobjekts erhält.

Die Interaktivität der Lernobjekte (Schulmeister 2003), die weder mit der Interaktion mit der Schinttstelle noch mit der Navigation verwechselt werden sollte, bezieht sich auf die

- die Manipulation der Repräsentationsform

- die Manipulation des Inhalts

- die eigene Konstruktion von Wissen

- und die Rückmeldung vom Lernobjekt selbst.

Zusätzlich zur Rückmeldung vom Lernobjekt sollte es Rückmeldungen an den Lernenden in offenen Lernumgebungen geben, die von Tutoren und Lehrenden auf kommunikativem Wege erteilt werden, in Chats oder in Foren, und diese Form der Rückmeldung kann sich auf folgende Sachverhalte beziehen: 
- die Orientierung des Lernenden, die Navigation und den Umgang mit den Lernobjekten

- Propädeutik, Heuristik und Lernorganisation

- die wissenschaftliche Argumentation und Fragen der Methodologie

- den kognitiven Gehalt wissenschaftlicher Themen \& Theorien

- Fragen der Motivation

- psychosoziale Faktoren des Individuums, der Lernumwelt und der Lerngemeinschaft.

\section{Literatur}

Barrows, H.S./Pickell, G.C.: Developing clinical problem-solving skills. A guide to more effective diagnosis and treatment. London et al. 1991.

Bruner, J.S.: The Act of Discovery. In: Harvard Educational Review 31 (1961), S. 21-32.

Bundesassistentenkonferenz (Hrsg.): Forschendes Lernen - wissenschaftliches Prüfen (Schriften der BAK 5). Bonn 1970.

Cognition and Technology Group at Vanderbilt: Designing Learning Environments That Support Thinking: The Jasper Series as a Case Study. In: Duffy, T.M./ Lowyck, J./ Jonassen, D.H. (eds): Designing Environments for Constructive Learning. (NATO ASI Series. Series F: Computer and Systems Sciences; 105) Berlin/Heidelberg 1993, S. 9-36.

Collins, A.: Cognitive Apprenticeship and Instructional Technology. In: Idol, L. / Jones, B.F. (eds): Educational Values and Cognitive Instruction: Implications for Reform. Hillsdale, NJ u.a.1991, S. 121-138.

de Jong, T./ Njoo, M.: Learning and Instruction with Computer Simulations: Learning Processes Involved. In: De Corte, E. et al (eds): Computer-Based Learning Environments and Problem Solving. (NATO ASI Series. Series F: Computer and Systems Sciences; 84) Berlin/Heidelberg 1992, S. 411-427.

Elen, J./ Lowyck, J./van den Berg, B.: Virtual University? Will Learning Benefit? - In: Ortner, G.E. / Nickolmann, F. (Hrsg.): Socio-Economics of Virtual Universities. Weinheim 1999.

Goodyear, P./ Tait, K.: Learning with Computer-Based Simulations: Tutoring and Student Modelling Requirements for an Intelligent Learning Advisor. In: Carretero, M. et al (eds): Learning and Instruction: European Research in an International Context. Vol. 3. Oxford 1991, S. 463-481.

Gräsel, C./ Mandl, H./ Prenzel, M.: Die Förderung diagnostischen Denkens durch fallbasierte Computerlernprogramme in der Medizin. In: Glowalla, U./ Schoop, E. (Hrsg.): Hypertext und Multimedia. Neue Wege in der computerunterstützten Aus- und Weiterbildung. Berlin/Heidelberg 1992, S. 323-331.

Jonassen, D.H.: Toward a design theory of problem solving. Educational Technology: Research \& Development, 48 (2000/4), S. 63-85.

Lave, J. Wenger, E.: Situated Learning: Legitimate Peripheral Participation. Cambridge 1991.

Lowyck, J./ Elen, J.: Transitions in the Theoretical Foundation of Instructional Design. In: Duffy, T.M./Lowyck, J./ Jonassen, D.H. (eds): Designing Environments for Constructive Learning. (NATO ASI Series. Series F: Computer and Systems Sciences; 105) Berlin/Heidelberg 1993, S. 213-229.

Metzger, Ch./ Schulmeister, R,/ Zienert, H.: Die Firma. Gebärdensprache Do It Yourself. Seedorf 2000.

Metzger, Ch./ Schulmeister, R./ Zienert, H.: Die Firma. Gebärdensprache Interaktiv. Seedorf 2003. 
Millis, B.J./ Cottel Jr., Ph.G. (eds): Cooperative Learning for Higher Education Faculty. o.O. 1998. Neber, H. (Hrsg.): Entdeckendes Lernen. Weinheim/Basel 1975.

Palincsar, A.S./ Brown, A.L.: Reciprocal Teaching of Comprehension-Fostering and ComprehensionMonitoring Activities. In: Cognition \& Instruction 1 (1984), S. 117-175.

Popescu, H. / Hong, S,-E. / Schmaling, C. / Hanke, Th. / Schulmeister, R.: Signing Tutor. Hamburg 2002, ViSiCAST deliverable 2-3.

Schmidt, H.G. et al. (eds): New Directions for Medical Education. Problem-based Learning and Community-oriented Medical Education. Berlin, Heidelberg, New York, London, Paris, Tokyo 1989.

Schulmeister, R.: Zur Komplexität Problemorientierten Lernens. In: Asdonk, J u.a. (Hrsg.): Bildung im Medium der Wissenschaft. Zugänge aus Wissenschaftspropädeutik, Schulreform und Hochschuldidaktik. Weinheim 2002, S. 185-201.

Shulman, L.S. / Keislar, E.R. (eds): Learning by Discovery: A Critical Appraisal. Chicago 1966.

Winn, W.: A Constructivist Critique of the Assumptions of Instructional Design. In: Duffy, T.M./ Lowyck, J./ Jonassen, D.H. (eds): Designing Environments for Constructive Learning. (NATO ASI Series. Series F: Computer and Systems Sciences; 105) Berlin/Heidelberg 1993, S. 189-212. 